
resolution by femtosecond laser filament

SUBJECT AREAS:

ULTRAFAST PHOTONICS

TERAHERTZ OPTICS

Received

14 October 2013

Accepted

8 January 2014

Published

24 January 2014

Correspondence and requests for materials should be addressed to W.W.L. (liuweiwei@ nankai.edu.cn) or Y.C. (ya.cheng@siom.ac.

\section{in air}

Jiayu Zhao', Wei Chu' ${ }^{2}$ Lanjun Guo' , Zhi Wang' ' Jing Yang' ', Weiwei Liu', Ya Cheng² \& Zhizhan Xu²

'Institute of Modern Optics, Nankai University, Key Laboratory of Optical Information Science and Technology, Ministry of Education, Tianjin 300071, China, ${ }^{2}$ State Key Laboratory of High Field Laser Physics, Shanghai Institute of Optics and Fine Mechanics, Chinese Academy of Sciences, Shanghai 201800, China.

Terahertz (THz) imaging provides cutting edge technique in biology, medical sciences and non-destructive evaluation. However, due to the long wavelength of the $\mathrm{THz}$ wave, the obtained resolution of $\mathrm{THz}$ imaging is normally a few hundred microns and is much lower than that of the traditional optical imaging. We introduce a sub-wavelength resolution $\mathrm{THz}$ imaging technique which uses the $\mathrm{THz}$ radiation generated by a femtosecond laser filament in air as the probe. This method is based on the fact that the femtosecond laser filament forms a waveguide for the $\mathrm{THz}$ wave in air. The diameter of the $\mathrm{THz}$ beam, which propagates inside the filament, varies from $20 \mu \mathrm{m}$ to $50 \mu \mathrm{m}$, which is significantly smaller than the wavelength of the $\mathrm{THz}$ wave. Using this highly spatially confined $\mathrm{THz}$ beam as the probe, $\mathrm{THz}$ imaging with resolution as high as $20 \mu \mathrm{m}(\sim \lambda / 38$ at $0.4 \mathrm{THz})$ can be realized.

esolution enhancement of terahertz (THz) imaging is one of central concerns in the THz science and technology research. Because of the used long wavelength $\left(\lambda_{1 \mathrm{THz}}=300 \mu \mathrm{m}\right)$, THz imaging's resolution is generally in the scale of millimetre. This constitutes a major obstacle for the application of THz imaging in bio-medical diagnosis and semiconductor device inspection since the characteristic scales of the interested samples are often much smaller than the wavelength of the $\mathrm{THz}$ wave ${ }^{1-3}$. Different approaches have been implemented to optimize the $\mathrm{THz}$ imaging resolution. $\mathrm{M}$. Tounouchi and his collaborators have developed a laser THz emission microscopy (LTEM) system ${ }^{3}$. The system detects the actively excited $\mathrm{THz}$ signal by focusing femtosecond laser beam on the semiconductor device. The resolution of the system is determined by the focus size of the laser beam and is about several microns. Another type of method relies on the analogues of the scanning near-field optical microscopy (SNOM). A so-called dynamic aperture produced by a gating laser beam and a tapered metal tip with a small exit aperture have been used by X.-C. Zhang's group and S. Hunsche et al., respectively, to limit the size of the $\mathrm{THz}$ wave illuminated on the sample ${ }^{4,5}$. In both cases, the resulted resolution is a few tens microns. H.-T. Chen et al. further applied the apertureless SNOM by substituting the aperture with a sharp tip ${ }^{6}$. The reported resolution by this means is $150 \mathrm{~nm}$. However, when applying SNOM, only small portion of the THz wave energy is used. For example, as mentioned in Ref. 6 , it is about $0.5 \%$.

Here, we report on a novel sub-wavelength $\mathrm{THz}$ imaging method via the femtosecond laser filament in air, which refers to the plasma channel created by a femtosecond laser pulse ${ }^{7}$. One of the impressive characteristics of this novel THz source is its exceptional high field strength. The reported maximum electric field strength of the THz pulse generated by the filamentation has reached $4.4 \mathrm{MV} / \mathrm{cm}$ with a compact table-top infrared femtosecond laser setup ${ }^{8}$. In order to further increase the strength of the $\mathrm{THz}$ wave, two-colour laser field pumping scheme is often chosen in the practice ${ }^{9-11}$. This new type THz source has received intense research interest recently ${ }^{7-16}$. Particularly, because via appropriate optical adjustment, one may locate the filament at a remote distance as far as a few hundred metres, THz remote sensing has been proposed by using this technique to overcome the strong diffraction and the energy attenuation due to water vapour absorption in air $^{10,11}$.

In the present work, we have investigated the variation of the THz beam diameter along the filament during a two-colour laser pumping experiment. The results indicate that the $\mathrm{THz}$ beam diameter varies between $20 \mu \mathrm{m}$ and $50 \mu \mathrm{m}$ in the region where a filament, i.e., a plasma column, exists. By using this strongly confined THz beam as the illumination source, we demonstrate that a scanning $\mathrm{THz}$ imaging setup with sub-wavelength resolution down to $20 \mu \mathrm{m}$ is promising. Since the illuminating THz beam's diameter is inherently much smaller than the peak wavelength of the generated $\mathrm{THz}$ pulse $(\sim 0.4 \mathrm{THz})$, neither a limiting aperture nor a scattering metal tip 


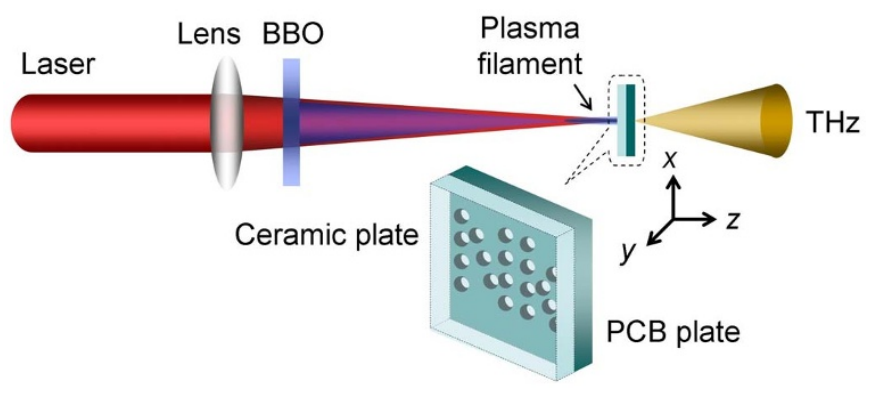

Figure 1 Experimental setup for sub-wavelength resolution $\mathrm{THz}$ imaging. A repetition rate of $1 \mathrm{kHz}$, central wavelength of $800 \mathrm{~nm}$ and duration of $50 \mathrm{fs}$ Ti: sapphire laser pulse with energy of $1 \mathrm{~mJ} /$ pulse was focused by a $f=30 \mathrm{~cm}$ lens in air. The focused pump beam passed a 0.1 mm-thick Type I $\beta$-barium borate (BBO) crystal, creating plasma at the focus. $\mathrm{THz}$ emission from this plasma filament is utilized as the probe of $\mathrm{THz}$ imaging and measured by a standard EOS (electric-optic sampling) setup. The object to be imaged is a PCB (printed circuit board) plate with multiple through-holes drilled on the plate, inserted in the middle of the filament. An additional ceramic plate was put in front of the PCB plate to terminate the laser beam and exclude the laser ablation on the sample.

suggested in the conventional THz-SNOM is necessary. The sample does not need to be an active medium being able to generate $\mathrm{THz}$, neither. Therefore, better performance in signal to noise ratio (SNR) could be prospected for our technique.

\section{Results}

THz sub-wavelength imaging by femtosecond laser filament. Fig. 1 schematically shows the experimental setup. Two-colour laser field $(800 \mathrm{~nm}+400 \mathrm{~nm})$ was focused in $\operatorname{air}(f=30 \mathrm{~cm})$. A filament was then created at the vicinity of the focus. A single cycle $\mathrm{THz}$ pulse will be generated, and it is highly forwardly directional. The generated THz pulse was detected by a standard electric-optic sampling (EOS) setup, which has been described in detail elsewhere ${ }^{12}$. Since the beam diameter would be larger after the filament has ended ${ }^{13}$, subwavelength resolution $\mathrm{THz}$ imaging was carried out by inserting a sample in the middle of the filament. The sample used in our experiment was a PCB (printed circuit board) plate. Multiple through-holes were drilled on the plate. The thickness of the PCB plate is about $1.5 \mathrm{~mm}$. Just in front of the PCB plate, a ceramic plate with a thickness of $0.8 \mathrm{~mm}$ was placed, in touch with the PCB plate. Because the ceramic plate is highly resistible to the high intensity femtosecond laser, it terminates the filament and excludes the laser damage of the sample. No laser transmitting or noticeable damage on the ceramic plate could be found during or after the experiment. The $\mathrm{THz}$ power transmission of the ceramic plate is about $50 \%$ (see Methods). The transmission could be further increased by using a thinner ceramic plate. On the other hand, the PCB plate has poor transmission of $\mathrm{THz}$ wave. Mainly the $\mathrm{THz}$ energy passing through the holes could be detected by EOS setup. The PCB plate and the ceramic plate were fixed on a two-axis translation stage and the $\mathrm{THz}$ image of the holes on the PCB plate was taken by moving the stage in the $x-y$ plane ( $z$ axis is defined as the laser propagation direction). The step sizes were $100 \mu \mathrm{m}$ and $100 \mu \mathrm{m}$ along $x$ and $y$ axes, respectively. Note that full $\mathrm{THz}$ temporal waveforms have been recorded for each position.

Fig. 2a illustrates the image of the multiple holes under optical microscope (resolution: $5 \mu \mathrm{m}$ ). The diameter of each hole is about $600 \mu \mathrm{m}$. The holes form two characters of "NK", abbreviation for NanKai. The corresponding scanning $\mathrm{THz}$ image is displayed in Fig. 2b. Comparing Fig. 2a and 2b, no significant blurring effect could be noticed. In order to further highlight this trend, Fig. $2 \mathrm{c}$ plots the spatial profiles of a series of representative holes retrieved from both pictures along the green dotted line. The boundary of the hole identified in the THz imaging (blue solid squares) is as distinct as that of the optical imaging. Reminding ourselves that the peak wavelength of the $\mathrm{THz}$ pulse generated in our experiment is about $750 \mu \mathrm{m}$, which is even larger than the size of the holes on the PCB plate. However, Fig. 2 indicates that the minimum resolvable structure by $\mathrm{THz}$ imaging is less than $100 \mu \mathrm{m}$. For example, according to the optical image (Fig. 2a), three hole pitches pointed by arrow A, $\mathrm{B}$ and $\mathrm{C}$ have characterized widths of about $60 \mu \mathrm{m}, 80 \mu \mathrm{m}$ and $75 \mu \mathrm{m}$, respectively. And they can be clearly resolved by the $\mathrm{THz}$ imaging (Fig. 2b). Hence, the resolution of the obtained $\mathrm{THz}$ image by our method is much smaller than the $\mathrm{THz}$ pulse wavelength. It is interesting to mention that most of the previous $\mathrm{THz}$ sub-wavelength imaging experiments are performed on the surface of the targets ${ }^{3,6}$. In our experiment, the thickness of the PCB plate, which was used as the imaging target (please see Fig. 2), is about $1.5 \mathrm{~mm}$. It hints that the effective length within which the diameter of the $\mathrm{THz}$ beam can be maintained to be more or less the same is about $1.5 \mathrm{~mm}$.

Resolution of THz imaging (Diameter of $\mathrm{THz}$ beam emission from femtosecond laser filament). Efforts have been made to quantify the achievable resolution of the $\mathrm{THz}$ imaging by using the filamentation. The knife-edge method (KE) was applied to measure the $\mathrm{THz}$ beam diameter, which determines the $\mathrm{THz}$ imaging resolution in our experiment. During the experiment, we found that a PCB plate is indeed a good material to be used to cut the $\mathrm{THz}$ beam for two
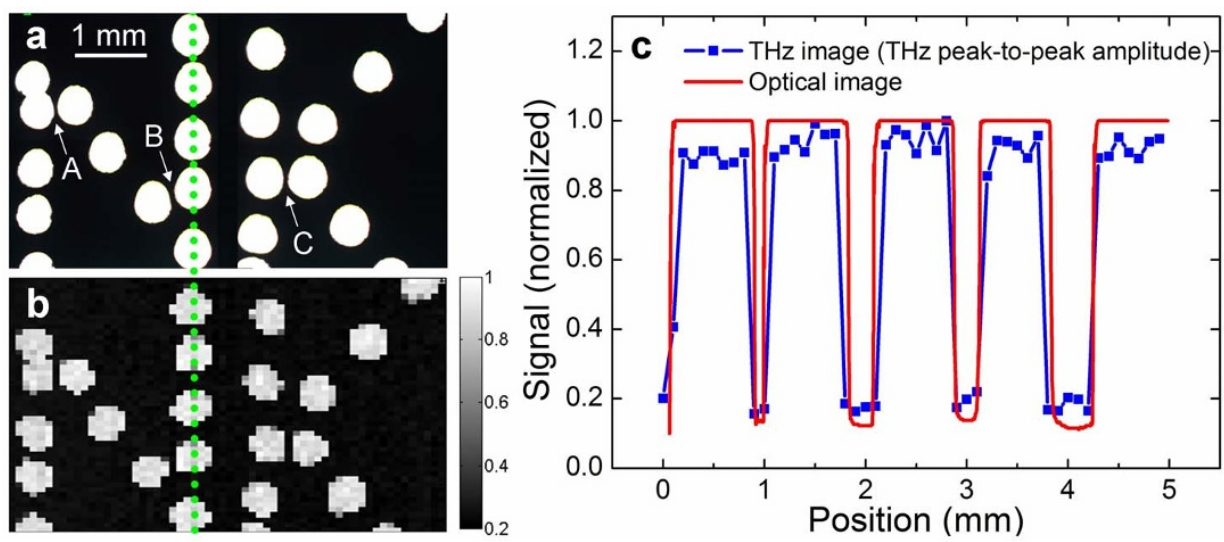

Figure $2 \mid$ Comparing THz image and optical microscope image. (a) Optical microscope image of multiple through-holes on PCB (printed circuit board) plate. Each hole is about $600 \mu \mathrm{m}$ in size. Arrow A, B and C point at three hole pitches with characterized widths of about $60 \mu \mathrm{m}, 80 \mu \mathrm{m}$ and $75 \mu \mathrm{m}$, respectively. (b) THz image of multiple through-holes on PCB plate. (c) Spatial profiles of a series of representative holes retrieved from (a) and (b) along the green dotted line, as red line and blue solid squares, respectively. 


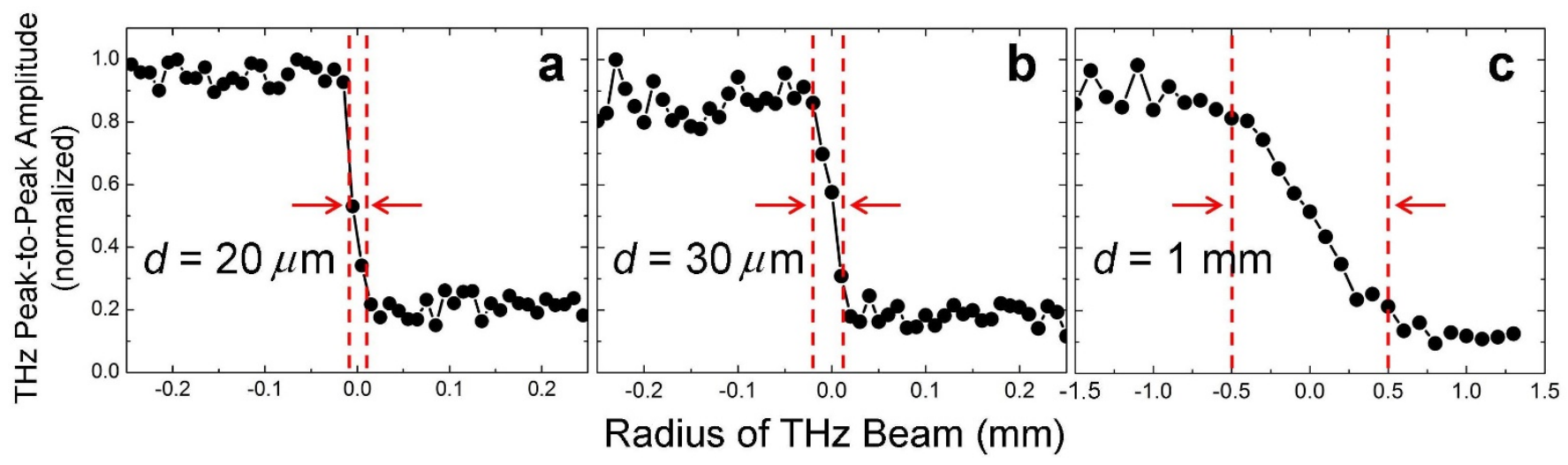

Figure $3 \mid$ Three representative knife-edge measurements of THz beam diameter $\boldsymbol{d}$. (a) $d=20 \mu \mathrm{m}$ at $z=0 \mathrm{~mm}$. (b) $d=30 \mu \mathrm{m}$ at $z=3 \mathrm{~mm}$. (c) $d=$ $1 \mathrm{~mm}$ at $z=6 \mathrm{~mm}$. $z$ axis corresponds to the laser propagation direction and $z=0$ is defined as the starting position where significant THz signal was able to be detected.

reasons: it is also highly resistible to the ablation of the intense femtosecond laser and has low transmission for $\mathrm{THz}$ wave $(\sim 4 \%$ power transmission). This time, a PCB plate was solely used and was scanned across the filament to perform the knife-edge measurement at different positions $z$. Note that $z=0$ corresponds to the starting position where significant $\mathrm{THz}$ signal was able to be detected. Three representative measurements are demonstrated in Fig. 3 for $z=0 \mathrm{~mm}, 3 \mathrm{~mm}$ and $6 \mathrm{~mm}$, respectively. All the three plots have been normalized to their maxima. The $\mathrm{THz}$ beam diameters $d$ interpreted from Fig. 3 are $d=20 \mu \mathrm{m}$ for $z=0 \mathrm{~mm}$, $d=30 \mu \mathrm{m}$ for $z=3 \mathrm{~mm}$, and $d=1 \mathrm{~mm}$ for $z=6 \mathrm{~mm}$, respectively. The obtained $\mathrm{THz}$ beam diameters $d$ as a function of $z$ is depicted in Fig. 4. THz beam diameter is initially $20 \mu \mathrm{m}$ at $z=0$. It slowly increases to $50 \mu \mathrm{m}$ at $z=5 \mathrm{~mm}$. Then a steep increase occurs between $z=5 \mathrm{~mm}$ and $z=6 \mathrm{~mm}(d=1 \mathrm{~mm})$. Afterwards, the $\mathrm{THz}$ beam diameter continuously increases to $6 \mathrm{~mm}$ when $z=$ $24 \mathrm{~mm}$. The results shown in Fig. 4 imply that the filament is sensitive to the PCB plate in our experiment. It could be attributed to the fact that when the PCB plate approaches the filament, it will block the energy reservoir, namely, the low intensity energy background surrounding the filament. Since the energy reservoir is the source to maintain the generation of the filament, the filament

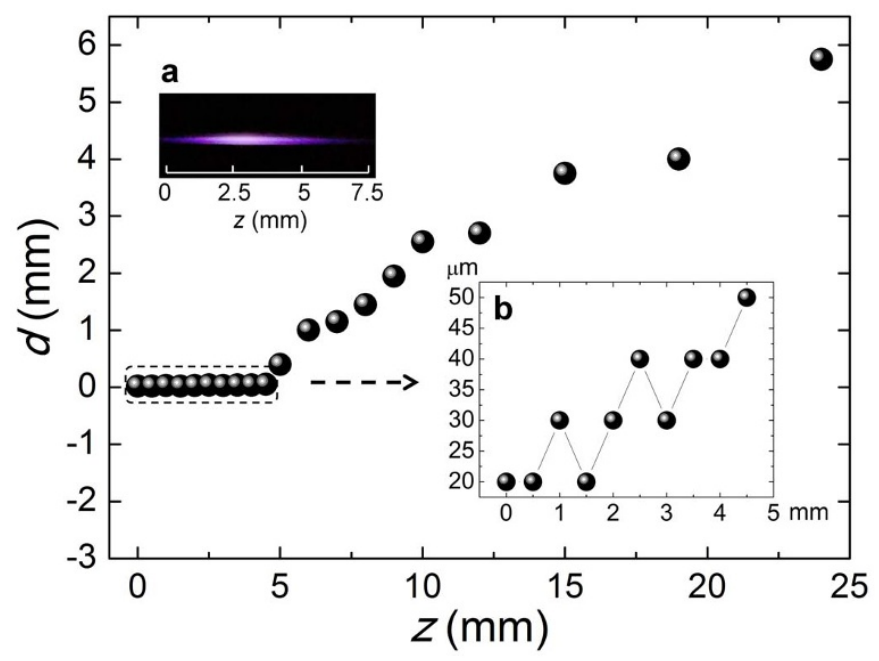

Figure $4 \mid \mathrm{THz}$ beam diameter $\boldsymbol{d}$ as a function of $z$. The insets (a) and (b) are side image of plasma filament and partially enlarged detail of $d$ measurement results from $z=0 \mathrm{~mm}$ to $z=5 \mathrm{~mm}$. $z$ axis corresponds to the laser propagation direction and $z=0$ is defined as the starting position where significant $\mathrm{THz}$ signal was able to be detected. confinement will be terminated when the PCB plate approaches the filament ${ }^{14}$.

Considering that $\mathrm{THz}$ pulse generated in our experiment will consist of broadband frequencies ${ }^{9-13,15-17}$, we have also studied the frequency dependence of the THz beam diameters. Thus, fast Fourier transformations (FFT) have been performed for all the recorded $\mathrm{THz}$ waveforms. Based on the results, two representative frequencies, namely peak frequency $f_{p}$ and mean frequency $f_{m}$ have been investigated. The mean frequency was determined according to:

$$
f_{m}=\frac{\sum_{f=0}^{\infty} S(f) \times f}{\sum_{f=0}^{\infty} S(f)},
$$

where $f$ denotes the frequency and $S(f)$ corresponds to the amplitude of the $\mathrm{THz}$ spectrum. The variations of the peak frequency and the mean frequency are depicted in Fig. 5a with respect to the propagation distances. Fig. 5a indicates that the peak frequency and the mean frequency are approximately $0.4 \mathrm{THz}$ and $0.63 \mathrm{THz}$, respectively. Then, the knife-edge measurements demonstrated by Fig. 3 were carried out for the amplitudes of the $\mathrm{THz}$ spectra at these two frequencies. The outcome is illustrated in Fig. 5b. Similar trends like Fig. 4 could be seen in Fig. $5 \mathrm{~b}$ for both $f_{p}$ and $f_{m}$. According to Fig. $5 \mathrm{~b}$, a sub-wavelength resolution of $\lambda / 38$ could be achieved at $0.4 \mathrm{THz}$.

THz wave guiding by femtosecond laser filament in air. What Fig. 4 impresses us most is that the $\mathrm{THz}$ pulse energy is spatially constrained inside a space which is much smaller than the wavelength. The jump of the curve in Fig. 4 taking place between $z=5 \mathrm{~mm}$ and $z=6 \mathrm{~mm}$ gives a hint that the $\mathrm{THz}$ wave is in fact strongly guided from $z=$ $0 \mathrm{~mm}$ to $z=5 \mathrm{~mm}$. Coincidentally, this region superposes with the zone where significant plasma, i.e. a filament, was produced during the filamentation. Fig. 4a presents a picture of the filament created in our experiment. It was taken by a digital camera set perpendicularly to the $z$ axis. In view of the overlapped locations of the $\mathrm{THz}$ wave guiding and plasma generation, we have reason to believe that a $\mathrm{THz}$ waveguide is created inside the filament. Note that wave guiding by plasma filaments has been proposed and demonstrated at microwave frequencies ${ }^{18-22}$. Our results imply that similar guiding phenomenon may also occur at $\mathrm{THz}$ band. It is this waveguide that strongly confines the $\mathrm{THz}$ energy into a region with only a few ten microns in diameter. And this phenomenon makes a novel subwavelength $\mathrm{THz}$ imaging technique feasible as we have demonstrated.

In order to confirm that creation of a $\mathrm{THz}$ waveguide by the filamentation, we have estimated the plasma density profile created by the two-colour laser field and calculated the corresponding 

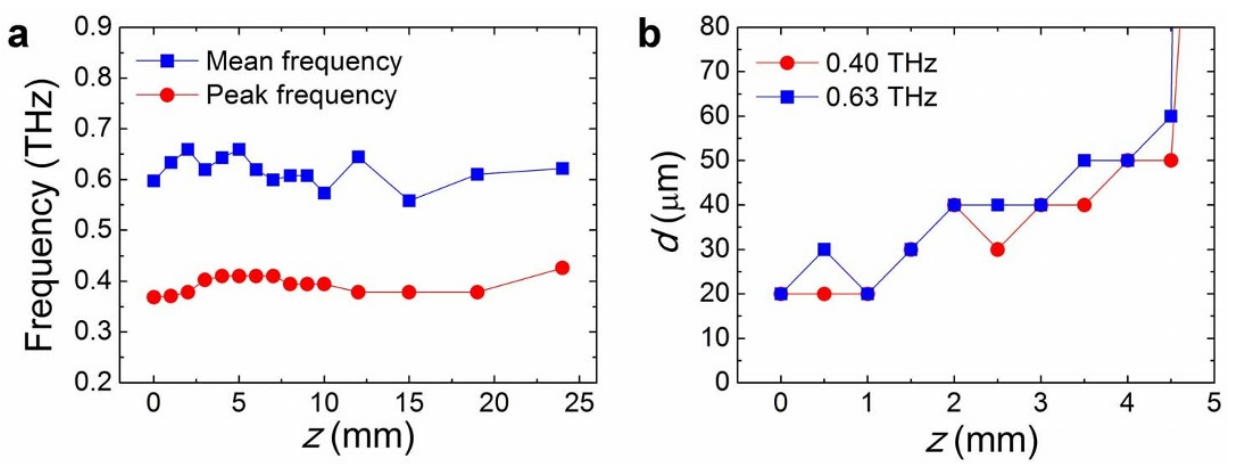

Figure $5 \mid$ Frequency dependence of the THz beam diameters $\boldsymbol{d}$. (a) Variations of peak frequency $\left(f_{p}\right.$, red solid circles) and mean frequency $\left(f_{m}\right.$, blue solid squares) with respect to the propagation distances $z$. (b) THz beam diameters $d$ as a function of $z$ at $f_{p}$ and $f_{m}$, in both cases the trends are similar to Fig. 4.

refractive index distribution as well. Following Ref. 15, we take the peak intensity of the fundamental beam and the second harmonic beam at focus as $I_{\omega}=1 \times 10^{14} \mathrm{~W} / \mathrm{cm}^{2}$ and $I_{2 \omega}=2 \times 10^{13} \mathrm{~W} / \mathrm{cm}^{2}$, respectively. Assuming two-colour pulses are in phase $\left(\phi_{\omega-2 \omega}=0\right)$, the obtained peak plasma density is $N_{\mathrm{e}}=2.34 \times 10^{17} \mathrm{~cm}^{-3}$ according to the static tunnelling ionization rate described in Ref. 9 and Methods. Then the plasma distribution at the focus is considered as a Gaussian whose full width at half maximum (FWHM) is about $50 \mu \mathrm{m}$ according to the result of side CCD image of the filament (not shown). Therefore, the refractive index distribution at the focus is given by

$$
n_{T H z}=\operatorname{Re}\left(\sqrt{1-\frac{\omega_{p}^{2}}{\Omega^{2}-i v \Omega}}\right),
$$

where $\omega_{p}$ indicates plasma frequency (in SI units):

$$
\omega_{p}=\sqrt{\frac{e^{2}}{m_{e} \varepsilon_{0}} N_{e}} .
$$

$N_{\mathrm{e}}$ denotes the number density of electrons, $e$ represents the electric charge, $m_{\mathrm{e}}$ indicates the effective mass of the electron and $\varepsilon_{0}$ is the permittivity in vacuum. $v \sim 1 \mathrm{THz}$ corresponds to the typical electron collision frequency inside filament ${ }^{16}$. The calculated radial distributions of $N_{e}$ and $n_{0.4} \mathrm{THz}$ are shown in Fig. 6, as blue line and red line, respectively. One can see a refractive index dip appears at the vicinity of $r=55 \mu \mathrm{m}$.

Then, we calculate the $\mathrm{THz}$ wave eigenmodes given by the refractive index distribution mentioned above. The calculation was carried out by the full-vector finite-element method (FEM) with the commercial software COMSOL Multiphysics. In our FEM model, quadratic triangular elements with a maximum size $1 \mu \mathrm{m}$ and the perfectly matched layers (PMLs) at the boundary of air area were applied. The doublet degenerated modes localized in the filament area are found in our simulation. Fig. 7 shows the intensity profiles ( $z$-component of the Poynting vector) and electric field vector of these modes at $0.2 \mathrm{THz}, 0.4 \mathrm{THz}$ and $0.6 \mathrm{THz}$, respectively. Since the fields are identical after a rotation of $\pi / 2$ radian, only one of the doublet degenerated modes is illustrated. As shown in Fig. 7, the intensity fields are localized in a circular region with diameters of about $130 \mu \mathrm{m}, 110 \mu \mathrm{m}$ and $105 \mu \mathrm{m}$ at $0.2 \mathrm{THz}, 0.4 \mathrm{THz}$ and $0.6 \mathrm{THz}$, respectively. The mode profiles given by Fig. 7 agree with recent reports that the emissions pattern in two-colour $\mathrm{THz}$ generation processes is a conical wave $\mathrm{i}^{13,23-25}$. Particularly, M. Clerici et al. have pointed out that the $\mathrm{THz}$ beam is Bessel shaped and the selfhealing properties of the Bessel beam would not play a significant role in KE experiment as demonstrated by Ref. 25. Fig. 7 also indicates that the electric field vector is nearly radial polarization for these modes. Note that the calculation is made for the focus, where the plasma density and diameter may be strongest. The actual diameters of $\mathrm{THz}$ mode fields could be smaller than $100 \mu \mathrm{m}$ along the filament. To compare with the refractive index distribution, the mode field intensity at $0.4 \mathrm{THz}$ along the white line in Fig. $7 \mathrm{~b}$ is also shown in Fig. 6 (black line). At $0.4 \mathrm{THz}$, the mode field is strongly confined in a sub-wavelength region, and the maximum light field is located at the refractive index dip.

It is worth mentioning that in our simulation scheme, the $\omega-2 \omega$ phase shift $\phi_{\omega-2 \omega}$ mainly affects the generated peak plasma density $N_{e}$. When $\phi_{\omega-2 \omega}=0, N_{e}$ is maximum; $N_{e}$ would reach a minimum value of $1.76 \times 10^{17} \mathrm{~cm}^{-3}$. Similar mode distribution can be obtained by assuming $N_{e}=1.76 \times 10^{17} \mathrm{~cm}^{-3}$ in our simulation. Therefore, the $\omega-2 \omega$ phase shift does not play a significant role in our simulation of the mode distribution.

\section{Discussion}

The calculation of Fig. 6 is made based on the assumption that the plasma density distribution follows Gaussian. However, it might not be totally fulfilled due to the strong space-time self-transformation occurred during the filamentation. This may explain the quantitative difference of the $\mathrm{THz}$ beam diameters shown in Fig. 4 and 6. Furthermore, since a conventional THz-TDS (time domain spectroscopy) setup is implemented as the detection method, a complete $\mathrm{THz}$ pulse waveform has to be acquired by scanning the optical delay

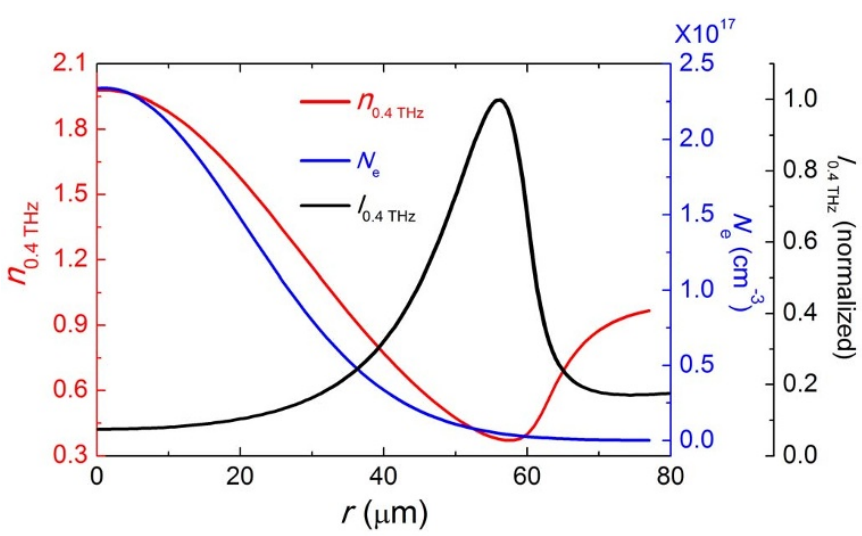

Figure 6 Distributions of plasma density $N_{e}$, refractive indices $n_{0.4} \mathrm{THz}$ and mode field intensity $I_{0.4} \mathrm{THz}$ as a function of $r . N_{e}$ distribution (blue line) is considered as a Gaussian, with FWHM (full width at half maximum) of $50 \mu \mathrm{m}$. The corresponding $n_{0.4 \mathrm{THz}}$ distribution (red line) has a minimum around $r=55 \mu \mathrm{m}$. Mode field intensity distribution $I_{0.4 \mathrm{THz}}$ (black line) is retrieved from Fig. $7 \mathrm{~b}$ along the white line and its maximum is also located at about $r=55 \mu \mathrm{m}$. 

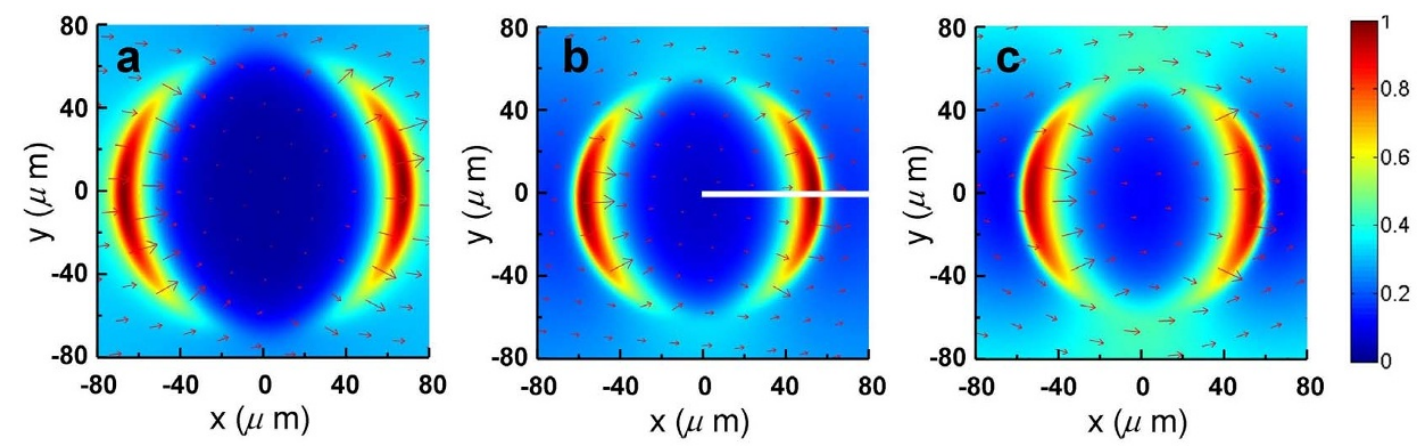

Figure $7 \mid$ Three typical THz eigenmodes around the filament area. One of the doublet degenerated modes at (a) $0.2 \mathrm{THz}$, (b) $0.4 \mathrm{THz}$ and (c) $0.6 \mathrm{THz}$ with $z$-component of the Poynting vector (colour) and electric vector (arrows). The mode field intensity along the white line in (b) is shown in Fig. 6 as black line.

in order to get the peak-to-peak amplitude of the THz pulse. Though both amplitude and phase information could be retrieved, it is quite time consuming. For example, a measurement of Fig. $2 \mathrm{~b}$ costs about 10 hours. If one was only interested in the power transmission, the measurement could be significantly speeded up by using $\mathrm{THz}$ power meter instead of TDS system.

Besides, M. Peccianti et al. have recently pointed out that special caution need to be paid to the $\mathrm{THz}$ field measurement by combining knife-edge method with time domain spectroscopy ${ }^{26}$. They have found that for a $\mathrm{THz}$ source with sub-wavelength dimension, the coupling between $x, y$ and $t$ coordinates has to be taken into account in order to reconstruct the THz field from KE + TDS system. In fact, M. Clerici et al. have demonstrated recently that for a conical beam, though the KE measurement could not resolve the spatial profile with fine details, it is still capable to give the essential information about the overall $\mathrm{THz}$ beam diameter ${ }^{25}$. In addition, Ref. 26 has suggested that the space-time coupling mainly affects the spatial symmetry of the measured $\mathrm{THz}$ beam profile, but does not alert the beam diameter significantly. Therefore, when the retrieving of the fine structure of the $\mathrm{THz}$ beam profile with high accuracy is not the central interest, $\mathrm{KE}+\mathrm{TDS}$ detection remains reliable to measure the overall beam diameter.

Furthermore, by using some other detection techniques, broader detection bandwidth could be achieved as compared with our TDS system $^{15,27-29}$. Thus, though the proof-of-principle of the sub-wavelength imaging method is reported in the present work for the low frequencies, the investigation about its application at high frequency range has been planned for future systemic study in terms of various experimental parameters, including pump laser energy ${ }^{30}$, pulse duration $^{31}$, laser polarization ${ }^{32}$, laser wavelength ${ }^{8}$, gas species ${ }^{16}$, gas pressure $^{33}$ and detection technique $e^{28-30,34,35}$, etc. Nevertheless, it could be foreseen that if adopting broad bandwidth detection methods, such as ABCD (Air-Breakdown Coherent Detection) ${ }^{27}$, sub-wavelength $\mathrm{THz}$ imaging technique demonstrated here may possess exceptional spectral analysis ability simultaneously.

In summary, we report on a novel technique to realize $\mathrm{THz}$ imaging with sub-wavelength resolution. Our method relies on the $\mathrm{THz}$ wave generated by a femtosecond laser filament in air. Because of the radial inhomogeneous distribution of refractive index given by the plasma density inside the filament, a $\mathrm{THz}$ waveguide is created. This plasma $\mathrm{THz}$ waveguide strongly confines the $\mathrm{THz}$ radiation into a region with a size of only a few tens micron. Using this filamentation generated $\mathrm{THz}$ source as a probe, the achievable $\mathrm{THz}$ imaging resolution can be as high as $20 \mu \mathrm{m}(\sim \lambda / 38$ at $0.4 \mathrm{THz})$ while retaining the exceptional high $\mathrm{THz}$ field strength achieved by the twocolour laser pumping experiment. Because of this advantage, it could be expected that without dramatic sacrifice the signal contrast, the $\mathrm{THz}$ imaging resolution could be further improved by inserting additional aperture with even smaller opening like SNOM.
Moreover, K. Wang et al. have recently demonstrated that an optical super-resolution of $40 \mu \mathrm{m}$ has been achieved when a target, which was placed at a distance of $4.5 \mathrm{~m}$ away from the laser output, was illuminated by the filament generated in $\operatorname{air}^{36}$. The combination of the two concepts, namely, the reported super-resolution $\mathrm{THz}$ imaging and the filament assisted remote detection ${ }^{10,11,36}$, may open a new way to realize sub-wavelength $\mathrm{THz}$ remote imaging.

\section{Methods}

Measurement of THz pulse waveform. The experimental setup is that a $1 \mathrm{kHz}$, $800 \mathrm{~nm}, 50 \mathrm{fs}$ Ti: sapphire laser pulse was split into two paths. One was the pump beam and the other was used as the probe of EOS. The energy of the pump beam was about $1 \mathrm{~mJ} /$ pulse and focused by a $f=30 \mathrm{~cm}$ lens. The thickness of the inserted Type I phase matching BBO crystal was $0.1 \mathrm{~mm}$. A filament was induced by the two-colour laser pulse at the focus. And the exiting $\mathrm{THz}$ pulse from the filament was first collimated by an off-axis parabolic mirror $(D=50 \mathrm{~mm}, f=100 \mathrm{~mm})$, then focused by another identical parabolic mirror onto a 1-mm-thick ZnTe crystal. Throughout the experiment, it has been carefully verified that the parabolic mirrors are bigger enough to collect all the emitted $\mathrm{THz}$ energy from the filament when performing $\mathrm{KE}$ method. The probe beam was combined with $\mathrm{THz}$ pulse by a Pellicle beam splitter, performing EOS measurement. Representative experimentally recorded $\mathrm{THz}$ electric field waveforms and the corresponding THz spectra are shown in Fig. 8a and 8b, respectively. It is worth mentioning that if the detection area of the EOS system is too small, when KE method is applied, the fraction of the power collected by the detector may vary with the position of the blade. In order to rule out this effect, the beam splitter was set between the focusing parabolic mirror and the $\mathrm{ZnTe}$ crystal in our experiment. Hence, only the THz beam was focused in the detection system, while the $800 \mathrm{~nm}$ probe beam retained its initial diameter $(\sim 8 \mathrm{~mm})^{12,37}$. In addition, the detector diameters of the two identical photodiodes used for balanced detection in our EOS system were $5 \mathrm{~mm}$, yielding to a large enough effective detection area to cover the whole focused $\mathrm{THz}$ beam inside the $\mathrm{ZnTe}$ crystal $^{25}$.

Calculation of plasma density. The ionization rate $R(t)$ could be commonly described by the static tunnelling model in the form of Ref. 9:

$$
R(t)=\frac{a}{A(t)} \exp \left[-\frac{b}{A(t)}\right],
$$

where

$$
A(t)=\frac{|\vec{E}(t)|}{E_{a}}
$$

is the electric field in atomic units and $E_{a}=k^{3} m^{2} e^{5} / \hbar^{4} \approx 5.14 \times 10^{11} \mathrm{~V} / \mathrm{m}$, $a=4 \omega_{a} r_{H}^{5 / 2}, b=(2 / 3) r^{3 / 2} \omega_{a}=k^{2} m e^{4} / \hbar^{3} \approx 4.16 \times 10^{16} / \mathrm{s} . \omega_{a}$ corresponds to the atomic frequency unit, $r_{\mathrm{H}}=U_{\mathrm{ion}} / U_{\mathrm{H}}$ indicates the ionization potential of the gas molecules under consideration relative to that of hydrogen $\left(U_{\mathrm{H}}=13.6 \mathrm{eV}\right)$ and $\kappa=$ $\left(4 \pi \varepsilon_{0}\right)^{-1}$. In the calculations, the air is considered as being composed of $78 \%$ nitrogen and $22 \%$ oxygen with $U_{\text {ion, } \mathrm{N} 2}=15.6 \mathrm{eV}$ and $U_{\text {ion, } \mathrm{O} 2}=12.1 \mathrm{eV}$. Hence, the electron density $N_{e}(t)$ in air is given by:

$$
d N_{e}(t)=d N_{e}(t)_{N_{2}}+d N_{e}(t)_{O_{2}},
$$

where

$$
\left\{\begin{array}{l}
d N_{e}(t)_{N_{2}}=R(t)_{N_{2}}\left[N_{0 N_{2}}-N_{e}(t)_{N_{2}}\right] d t \\
d N_{e}(t)_{O_{2}}=R(t)_{O_{2}}\left[N_{0 O_{2}}-N_{e}(t)_{O_{2}}\right] d t
\end{array}\right.
$$

$\mathrm{N}_{\mathrm{ON}_{2}}$ and $\mathrm{N}_{\mathrm{OO}_{2}}$ denote the neutral density of nitrogen and oxygen, respectively. Since the laser intensity at the focus is not precisely known, it is assumed that the peak 

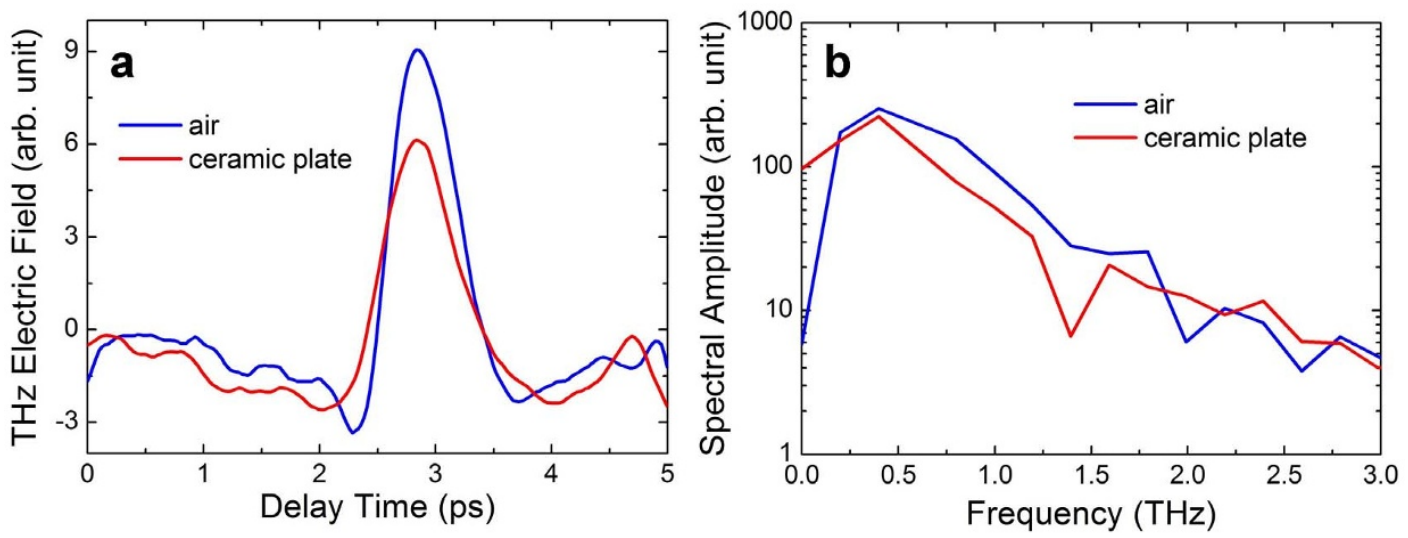

Figure 8 THz electric field waveforms and the corresponding THz spectra. (a) Measured THz pulse waveforms in air (blue line) and after transmission through the ceramic plate (red line). (b) The corresponding THz spectra, whose peak amplitudes are both at $0.4 \mathrm{THz}$.

amplitude of the fundamental beam $\left(\vec{E}_{1}(t)\right)$ in eq. $(5)$ is $2.75 \times 10^{8} \mathrm{~V} / \mathrm{cm}$, corresponding to a laser intensity of $I_{\omega}=1 \times 10^{14} \mathrm{~W} / \mathrm{cm}^{2}$ at the focus, calculated by

$$
I=\frac{|E|^{2}}{2 \eta}
$$

where $\eta=377 \Omega$ is the impedance. The peak amplitude of the second harmonic beam $\left(\vec{E}_{2}(t)\right)$ is assumed to be $1.23 \times 10^{8} \mathrm{~V} / \mathrm{cm}$, corresponding to $I_{2 \omega}=2 \times 10^{13} \mathrm{~W} / \mathrm{cm}^{2}$.

1. Tonouchi, M. Cutting-edge terahertz technology. Nat. Photonics 1, 97-105 (2007).

2. Zhang, X. C. Terahertz wave imaging: horizons and hurdles. Phys. Med. Biol. 47, 3667 (2002).

3. Inoue, R., Uchida, N. \& Tonouchi, M. Scanning probe laser terahertz emission microscopy system. Jpn. J. Appl. Phys. 45, L824-L826 (2006).

4. Chen, Q., Jiang, Z., Xu, G. X. \& Zhang, X. C. Near-field terahertz imaging with a dynamic aperture. Opt. Lett. 25, 1122-1124 (2000).

5. Hunsche, S., Koch, M., Brener, I. \& Nuss, M. C. THz near-field imaging. Opt. Commun. 150, 22-26 (1998).

6. Chen, H. T., Kersting, R. \& Cho, G. C. Terahertz imaging with nanometer resolution. Appl. Phys. Lett. 83, 3009-3011 (2003).

7. D Amico, C. et al. Conical forward $\mathrm{THz}$ emission from femtosecond-laser-beam filamentation in air. Phys. Rev. Lett. 98, 235002 (2007).

8. Clerici, M. et al. Wavelength scaling of terahertz generation by gas ionization. Phys. Rev. Lett. 110, 253901 (2013).

9. Roskos, H. G., Thomson, M. D., Kreß, M. \& Löffler, T. Broadband THz emission from gas plasmas induced by femtosecond optical pulses: From fundamentals to applications. Laser Photonics Rev. 1, 349-368 (2007).

10. Wang, T. J. et al. Toward remote high energy terahertz generation. Appl. Phys. Lett. 97, 111108 (2010).

11. Liu, J., Dai, J., Chin, S. L. \& Zhang, X. C. Broadband terahertz wave remote sensing using coherent manipulation of fluorescence from asymmetrically ionized gases. Nat. Photonics 4, 627-631 (2010).

12. Zhang, Y. et al. Non-radially polarized THz pulse emitted from femtosecond laser filament in air. Opt. Express 16, 15483-15488 (2008).

13. Zhong, H., Karpowicz, N. \& Zhang, X. C. Terahertz emission profile from laserinduced air plasma. Appl. Phys. Lett. 88, 261103-261103 (2006).

14. Liu, W. et al. Experiment and simulations on the energy reservoir effect in femtosecond light filaments. Opt. Lett. 30, 2602-2604 (2005).

15. Kim, K. Y., Glownia, J. H., Taylor, A. J. \& Rodriguez, G. Terahertz emission from ultrafast ionizing air in symmetry-broken laser fields. Opt. Express 15, 4577-4584 (2007).

16. Kim, K. Y., Taylor, A. J., Glownia, J. H. \& Rodriguez, G. Coherent control of terahertz supercontinuum generation in ultrafast laser-gas interactions. Nat. Photonics 2, 605-609 (2008).

17. Houard, A., Liu, Y., Prade, B., Tikhonchuk, V. T. \& Mysyrowicz, A. Strong enhancement of terahertz radiation from laser filaments in air by a static electric field. Phys. Rev. Lett. 100, 255006 (2008).

18. Chekalin, S. V. \& Kandidov, V. P. From self-focusing light beams to femtosecond laser pulse filamentation. Phys. Usp. 56, 123 (2013).

19. Askar'yan, G. A. Waveguide properties of a tubular light beam. Soviet Phys. JETP 28, 732 (1969).

20. Valuev, V. V. et al. Plasma channels formed by a set of filaments as a guiding system for microwave radiation. J. Commun. Technol. Elect. 55, 208-214 (2010).

21. Dormidonov, A. E., Valuev, V. V., Dmitriev, V. L., Shlenov, S. A. \& Kandidov, V. P. Laser filament induced microwave waveguide in air. In Proc. SPIE 6733, 67332S (2007)
22. Châteauneuf, M., Payeur, S., Dubois, J. \& Kieffer, J. C. Microwave guiding in air by a cylindrical filament array waveguide. Appl. Phys. Lett. 92, 091104-091104 (2008).

23. You, Y. S., Oh, T. I. \& Kim, K. Y. Off-axis phase-matched terahertz emission from two-color laser-induced plasma filaments. Phys. Rev. Lett. 109, 183902 (2012).

24. Blank, V., Thomson, M. D. \& Roskos, H. G. Spatio-spectral characteristics of ultra-broadband $\mathrm{THz}$ emission from two-colour photoexcited gas plasmas and their impact for nonlinear spectroscopy. New J. Phys. 15, 075023 (2013).

25. Clerici, M. et al. CCD-based imaging and 3D space-time mapping of terahertz fields via Kerr frequency conversion. Opt. Lett. 38, 1899-1901 (2013).

26. Peccianti, M. et al. Exact reconstruction of THz sub- $\lambda$ source features in knifeedge measurements. IEEE J. Sel. Top. Quantum Elect. 19, 8401211 (2013).

27. Dai, J., Xie, X. \& Zhang, X. C. Detection of broadband terahertz waves with a laserinduced plasma in gases. Phys. Rev. Lett. 97, 103903 (2006).

28. Wu, Q. \& Zhang, X. C. 7 terahertz broadband GaP electro-optic sensor. Appl. Phys. Lett. 70, 1784-1786 (1997).

29. Thomson, M. D., Blank, V. \& Roskos, H. G. Terahertz white-light pulses from an air plasma photo-induced by incommensurate two-color optical fields. Opt. Express 18, 23173-23182 (2010)

30. Oh, T. I. et al. Intense terahertz generation in two-color laser filamentation: energy scaling with terawatt laser systems. New J. Phys. 15, 075002 (2013)

31. Bartel, T., Gaal, P., Reimann, K., Woerner, M. \& Elsaesser, T. Generation of singlecycle $\mathrm{THz}$ transients with high electric-field amplitudes. Opt. Lett. 30, 2805-2807 (2005).

32. Xie, X., Dai, J. \& Zhang, X. C. Coherent control of THz wave generation in ambient air. Phys. Rev. Lett. 96, 075005 (2006).

33. Rodriguez, G. \& Dakovski, G. L. Scaling behavior of ultrafast two-color terahertz generation in plasma gas targets: energy and pressure dependence. Opt. Express 18, 15130-15143 (2010).

34. Karpowicz, N. et al. Coherent heterodyne time-domain spectrometry covering the entire "terahertz gap" Appl. Phys. Lett. 92, 011131-011131 (2008).

35. Babushkin, I. et al. Ultrafast spatiotemporal dynamics of terahertz generation by ionizing two-color femtosecond pulses in gases. Phys. Rev. Lett. 105, 053903 (2010).

36. Wang, K. et al. Remote sub-diffraction imaging with femtosecond laser filaments. Opt. Lett. 37, 1343-1345 (2012).

37. Zhang, Y. et al. Portraying polarization state of terahertz pulse generated by a twocolor laser field in air. Opt. Lett. 34, 2841-2843 (2009).

\section{Acknowledgments}

This work is financially supported by National Basic Research Program of China (2014CB339802, 2011CB808100) and National Natural Science Foundation of Chin (11174156). W.L. acknowledges the support of the open research funds of State Key Laboratory of High Field Laser Physics, Shanghai Institute of Optics and Fine Mechanics (SIOM).

\section{Author contributions}

W.L. and Y.C. planned and designed the experiments. J.Z., W.C. and L.G. performed the experiments. J.Z. and J.Y. analyzed the data. Z.W. performed the numerical simulation. Z.X. supervised the project. All authors participated in the discussion of the results and the writing of the manuscript.

\section{Additional information}

Competing financial interests: The authors declare no competing financial interests. 
How to cite this article: Zhao, J.Y. et al. Terahertz imaging with sub-wavelength resolution by femtosecond laser filament in air. Sci. Rep. 4, 3880; DOI:10.1038/srep03880 (2014). (c) (1) (2) This work is licensed under a Creative Commons AttributionBY ${ }_{\text {BC }}$ SA NonCommercial-ShareAlike 3.0 Unported license. To view a copy of this license, visit http://creativecommons.org/licenses/by-nc-sa/3.0 